\title{
Assessment of risk of obstructive sleep apnoea syndrome among patients attending a medical outpatient clinic in a tertiary health facility in South-West Nigeria
}

\author{
B O Adeniyi, ${ }^{1} \mathrm{MB}$ ChB, FWACP; O S Ilesanmi, ${ }^{2} \mathrm{MBBS}, \mathrm{MBA}$, MSc, FWACP; A M Adebayo, ${ }^{3} \mathrm{MBBS}$, FWACP; A O Kareem, ${ }^{2} \mathrm{MB}$ ChB; \\ O A Junaid, ${ }^{1}$ MB BS; A O Adeniji, ${ }^{4}$ MBBS, FWACS; G E Erhabor, ${ }^{5}$ MBBS, FWACP, FCCP, FRCP (Edin), FRCP (Lond)
}

\author{
${ }^{1}$ Department of Medicine, Federal Medical Centre, Owo, Ondo State, Nigeria \\ ${ }^{2}$ Department of Community Health, Federal Medical Centre, Owo, Ondo State, Nigeria \\ ${ }^{3}$ Department of Community Medicine, University College Hospital Ibadan, Oyo State, Nigeria \\ ${ }^{4}$ Department of ENT, Federal Medical Centre, Owo, Ondo State, Nigeria \\ ${ }^{5}$ Department of Medicine, Respiratory Unit, Obafemi Awolowo University, Ile-Ife, Osun State, Nigeria
}

Corresponding author: Bamidele Olaiya Adeniyi (delbis2003@yahoo.com)

Background. Obstructive sleep apnoea syndrome (OSAS) is associated with increased morbidity and mortality. However, there are few studies from Nigeria that have evaluated the prevalence of OSAS in medical outpatient clinics.

Objective. To determine the degree of the risk of OSAS among patients attending the medical outpatient clinic of the Federal Medical Centre, Owo, South-West Nigeria.

Methods. A cross-sectional survey was conducted among 208 medical outpatients using the Berlin questionnaire and the Epworth sleepiness scale (ESS).

Results. The mean (standard deviation) age of participants was 53.8 (16.5) years, 110 (52.9\%) were female, and $73.1 \%$ of participants had a high likelihood of sleep-disordered breathing (HSDB). Subjects with a high body mass index (BMI) were more likely to have sleep -disordered breathing when compared with those with a normal BMI $(26$ (96.3\%) v. $7(29.2 \%), p<0.001)$. The respondents with multiple primary morbidities were about 24 times more likely to have HSDB compared with respondents with a single morbidity $(p=0.009)$. The odds of having HSDB was 16 times greater in those with hypertension compared with patients with asthma (odds ratio 16,95\% confidence interval $3-83, p=0.001$ ). The Berlin questionnaire and the ESS were useful screening tools in the Nigerian setting.

Conclusion. In resource-poor settings in Africa, where there is an emphasis on screening and treating diseases of poverty, patients with medical conditions such as high BMI and hypertension should be screened for OSAS.

S Afr Resp J 2015;21(1):3-7. DOI:10.7196/SARJ.7740

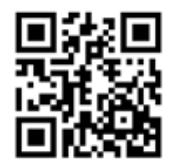

Obstructive sleep apnoea syndrome (OSAS) is increasingly becoming a disease of public health importance in Nigeria and Africa with longterm negative effects. It is a chronic illness that results from partial or complete collapse of the airway during sleep, and loud snoring is a common complaint by the patients or their partners. ${ }^{[1,2]}$ Some of the most common manifestations of OSAS include loud snoring, excessive daytime sleepiness (EDS) and breathing pauses during sleep. It is gradual in onset and the associated symptoms are mostly unrecognised by patients. ${ }^{[3]}$ OSAS contributes to long-term clinical consequences such as hypertension, cardiovascular disease and abnormalities in glucose metabolism. ${ }^{[4-6]}$

The prevalence of snoring among adults varies in different parts of the world from $19.3 \%$ to $52.3 \%$, while the risk of OSAS ranges from $16.8 \%$ to $33.3 \% \cdot{ }^{[7-9]}$ The prevalence of clinically suspected OSAS in a study done in Abuja, Nigeria was low at $1 \% \cdot{ }^{[9]}$ However, with increasing westernisation of diet, obesity, tendencies towards sedentary lifestyles and increasing prevalence of diabetes mellitus (DM) and hypertension, OSAS is becoming an increasing problem.

The laboratory overnight polysomnography (PSG) is the gold standard for diagnosis of OSAS but this is expensive and not commonly available. Resource constraints mean that many patients with a history of snoring or other symptoms of OSAS cannot easily be diagnosed in countries such as Nigeria. ${ }^{[10-14]}$ The practical assessment of patients at high risk of developing sleep-disordered breathing and OSAS includes use of validated questionnaires such as the Epworth Sleepiness Scale (ESS), the STOPBANG questionnaire, the Cleveland Sleep Habits Questionnaire and the Berlin questionnaire. These assessments are widely used to estimate and evaluate the likelihood of sleep-disordered breathing. Identifying those who are at risk of OSAS will help to determine who may require subsequent evaluation with more comprehensive tests. ${ }^{[10,15-18]}$

The majority of patients affected by OSAS are undiagnosed, and many physicians do not actively screen for this condition when evaluating other medical conditions that are associated with OSAS. ${ }^{[19]}$ 
This study was aimed at determining the risk of OSAS among patients attending a medical outpatient clinic in a tertiary health facility using the Berlin questionnaire and the ESS.

\section{Methods}

Study area

Owo is an ancient city located in the Owo local government area of Ondo State, South-West Nigeria. Agriculture (including fishing) constitutes the main occupation of the people. It is located about $350 \mathrm{~km}$ from Lagos State and $50 \mathrm{~km}$ from Akure, the state capital. The Federal Medical Centre (FMC) in Owo was a general hospital taken over by the Federal Government of Nigeria in 1989 and redesignated FMC with the aim of providing tertiary services in Ondo State. It is a 250-bed hospital with a staff of 1200 , of which doctors and nurses constitute about 500. It is the only tertiary hospital in Ondo state and provides primary, secondary and tertiary levels of care.

\section{Study design}

This was a descriptive cross-sectional study of adults attending the medical outpatient clinic of FMC, Owo.

\section{Study population}

The population sampled for this study included patients with common conditions attending the medical outpatient clinic, which was largely made up of patients with hypertension, DM, asthma and tuberculosis.

\section{Sample size determination}

The required sample size for this study was calculated using standard formulae for calculating minimum sample size for descriptive cross-sectional study.

Minimum sample size $=\mathrm{Za}^{2} p q / d^{2}$.

$\mathrm{Za}^{2}$ is the standard normal deviate corresponding to level of significance (usually 5\%). This was 1.96 .

$p$ is the prevalence of outcome of interest. A prevalence of high risk OSAS of $17.4 \%$ was used. ${ }^{[15]}$

$$
q \text { is } 1-p \text {. }
$$

$d$ is the level of precision ( 0.05 was used).

This sample size was calculated to be 220 .

However, only 208 patients were seen during the study period.

\section{Sampling method}

Consecutive patients who were not previously diagnosed with OSAS or sleep-disordered breathing were eligible for recruitment. Since the estimated minimum sample size was 220 subjects, and the clinic recorded an average of 80 patients per month, the study lasted from January to March 2014.

A semi-structured self-administered questionnaire was used for data collection. Medical doctors attending to patients at the outpatient clinic participated in questionnaire administration. The questionnaire was categorised into three sections using the objectives of the study as criteria: section A - sociodemographic characteristics; section B - ESS; section C - Berlin questionnaire for sleep evaluation. ESS score $<11$ was categorised as normal, while a score $\geq 11$ suggested EDS. Berlin questionnaire scores ranged from 0 to 3 , respondents with a score of 3 were categorised as having a high likelihood of sleep-disordered breathing (HSDB) while those scoring less were categorised as having a low likelihood of sleep-disordered breathing (LSDB). The patients' bed partner, when available, was asked about the presence of snoring, and the patients themselves were asked when there was no one who accompanied them to the hospital, or when those who accompanied them did not have such information.

\section{Data analysis}

The data obtained were analysed using the Statistical Package for Social Sciences (SPSS) for Windows evaluation version 21 (International Business Machines Corporation, USA). Data were presented using tables. Association between variables was assessed with $\chi^{2}$ test. Bivariate logistic regression analysis was used to determine predictors of high likelihood of sleepdisordered breathing. Level of significance was set at $p=0.05 \%$.

\section{Consent}

Permission to conduct the study was sought from each respondent before conducting the interview and a high level of privacy was ensured. Respondents were adequately informed that they had the right to decline participation or to withdraw from the study at any point in time. In addition, respondents were informed that refusal to participate in the study or withdrawal from it would not result in any penalty.

\section{Results}

There were 208 respondents, mean age (standard deviation (SD)) was 53.8 (16.5) years, $98(47.1 \%)$ males and $110(52.9 \%)$ were females. The most common diagnosis was hypertension in $66(38.2 \%)$. The sociodemographic characteristics and primary diagnoses of the respondents are summarised in Table 1. The Berlin questionnaire showed that 152 (73.1\%) had HSDB. Only 39 (18.8\%) had EDS using the ESS as shown in Table 2. Twenty-seven of the respondents (13\%) were obese and $54(26 \%)$ were overweight.

Among respondents aged $<44$ years, more than half (36 (62.1\%)) had a LSDB compared with 22 (37.9\%) who had HSDB $(p<0.001)$. Increasing body mass index (BMI) was associated with an increasing HSDB; respondents who were obese (26 (96.3\%))

Table 1. Sociodemographic characteristics and primary diagnosis of respondents

\begin{tabular}{ll}
\hline Variables & $\boldsymbol{n}(\%)$ \\
\hline Age (years) & \\
$<44$ & $58(27.9)$ \\
$44-64$ & $87(41.8)$ \\
$\geq 65$ & $63(30.3)$ \\
Sex & \\
Male & $98(47.1)$ \\
Female & $110(52.9)$ \\
Primary diagnosis $(N=173)$ & \\
Pulmonary tuberculosis & $41(23.7)$ \\
Hypertension & $66(38.2)$ \\
DM & $16(9.2)$ \\
Hypertension and DM & $34(19.7)$ \\
Asthma & $16(9.2)$
\end{tabular}

Table 2. Sleep evaluation of respondents using Berlin questionnaire and the Epworth sleepiness scale

\begin{tabular}{ll}
\hline Tool used & $\boldsymbol{n}(\%)$ \\
\hline Berlin sleep evaluation & \\
$\quad$ LSDB & $56(26.9)$ \\
HSDB & $152(73.1)$ \\
ESS & \\
$\quad$ Normal $<11$ & $169(81.3)$ \\
EDS $\geq 11$ & $39(18.8)$
\end{tabular}

LSDB = low likelihood of sleep-disordered breathing; HSDB = high likelihood of sleep-disordered breathing; EDS = excessive daytime sleepiness. 
had HSDB compared with the underweight respondents ( $7 \quad(29.2 \%), p<0.001)$. Table 3 shows the association between sociodemographic characteristics of the respondents and sleep-disordered breathing.

Table 4 shows the association between primary diagnosis and sleep-disordered breathing in respondents. Among respondents with multiple primary morbidity, 33(97.1\%) had HSDB compared with 103 (74.1\%) of respondents with single morbidity $(p=0.003)$. Of the hypertensive respondents, 63 (95.5\%) had HSDB while only 3 (4.5\%) had LSDB $(p<0.001)$.

The predictors of HSDB are shown in Table 5. Respondents aged 44 - 64 years were about 6 times more likely to have HSDB compared with those $<44$ years (odds ratio (OR) 5.6, 95\% confidence interval (CI) 1.5 20.7, $p=0.009$ ).

The likelihood of having HSDB increased with increasing BMI (OR 4.9, 95\% CI 1.1 21.9, $p=0.037$ ) in those with normal BMI (OR 13.3, 95\% CI $2.2-81.0, p=0.005)$ in overweight and markedly increased in obese respondents (OR 17.56, 95\% CI 1.4 215.5, $p=0.025)$ compared with those with underweight BMI. The respondents with multiple primary morbidities were about 24 times more likely to have HSDB compared with respondents having single morbidity (OR 23.7, 95\% CI $2.2-255.73, p=0.009$ ). The probability of having HSDB was much greater in respondents with hypertension compared with those with asthma (OR 15.6, CI $2.95-82.8, p=0.001$ ).

\section{Discussion}

OSAS is diagnosed using the standard laboratory overnight PSG, which is not commonly available, and is expensive and time and personnel intensive. This has led to OSAS being underdiagnosed, making accurate quantification of the health burden of untreated OSAS difficult, thus contributing to the occurrence of complications of OSAS, especially cardiovascular morbidities. ${ }^{[10-13]}$ The provision of validated questionnaires such as the Berlin questionnaire and the ESS thus serve as surrogates to identify patients with HSDB. ${ }^{[10,16-18]}$

The major finding of this study was that a significant number of patients who were being managed for chronic medical diseases in our tertiary hospital were at high risk of having OSAS. It was observed that most

\begin{tabular}{|c|c|c|c|c|}
\hline \multirow[b]{2}{*}{ Variables } & \multicolumn{2}{|c|}{ Sleep-disordered breathing } & \multirow[b]{2}{*}{$\chi^{2}$} & \multirow[b]{2}{*}{$p$-value } \\
\hline & LSDB $n(\%)$ & HSDB $n(\%)$ & & \\
\hline \multicolumn{5}{|c|}{ Age group in years } \\
\hline$<44$ & $36(62.1)$ & $22(37.9)$ & 0.505 & $<0.001$ \\
\hline $45-64$ & $11(12.6)$ & $76(87.4)$ & & \\
\hline$\geq 65$ & $9(14.3)$ & $54(85.7)$ & & \\
\hline \multicolumn{5}{|l|}{ Sex } \\
\hline Male & $28(28.6)$ & $70(71.4)$ & 0.256 & 0.613 \\
\hline Female & $28(25.5)$ & $82(74.5)$ & & \\
\hline \multicolumn{5}{|l|}{ BMI } \\
\hline Underweight & $17(70.8)$ & $7(29.2)$ & 0.387 & $<0.001$ \\
\hline Normal & $31(30.4)$ & $71(69.6)$ & & \\
\hline Overweight & $6(11.1)$ & $48(88.9)$ & & \\
\hline Obese & $1(3.7)$ & $26(96.3)$ & & \\
\hline
\end{tabular}

Table 4. Association between primary diagnosis and sleep-disordered breathing in respondents

\begin{tabular}{lllll}
\hline \multicolumn{2}{l}{ Sleep-disordered breathing } & & \\
Variables & LSDB $\boldsymbol{n}$ (\%) & HSDB $\boldsymbol{n}$ (\%) & $\chi^{\mathbf{2}}$ & $\boldsymbol{p}$-value \\
\hline $\begin{array}{l}\text { Number of primary morbidities } \\
\quad \text { Single }\end{array}$ & $36(25.9)$ & $103(74.1)$ & 8.564 & 0.003 \\
$\quad$ Multiple & $1(2.9)$ & $33(97.1)$ & & \\
Primary diagnosis & & & & \\
$\quad$ Pulmonary tuberculosis & $22(53.7)$ & $19(46.3)$ & 51.269 & $<0.001$ \\
Hypertension & $3(4.5)$ & $63(95.5)$ & & \\
DM & $3(18.8)$ & $13(81.3)$ & & \\
Hypertension and DM & $1(2.9)$ & $33(97.1)$ & & \\
Asthma & $8(50.0)$ & $8(50.0)$ & & \\
Daytime sleepiness & & & & \\
Non-excessive $<11$ & $44(26.0)$ & $125(74.0)$ & 0.361 & 0.548 \\
EDS $\geq 11$ & $12(30.8)$ & $27(69.2)$ & &
\end{tabular}

clinicians did not ask questions regarding snoring or EDS while attending to patients in the Medical Outpatients Department. ${ }^{[20]}$

In agreement with our findings, a study carried out at the university of Benin Teaching Hospital, Nigeria, showed that among a total of 102 medical outpatients with a mean age (SD) of 55.1 (13.6) years, the prevalence of OSAS risk among the respondents who were positive according to the Berlin questionnaire was 67 (65.7\%). The study also observed a statistically significant association between age group in years ( $p=0.01)$, BMI $(p<0.001)$, type of primary diagnosis $(p<0.001)$, and Berlin risk of OSAS. ${ }^{[20]}$
Our study showed a potentially high prevalence of OSAS among patients with chronic medical diseases as depicted by HSDB in the middle-aged and elderly groups using the Berlin questionnaire. However, the prevalence was low with the ESS. This may underscore the fact that the Berlin questionnaire has been documented to be clinically more sensitive and correlates significantly with the presence of OSAS among various populations. ${ }^{[10]}$ The ESS has been documented to be more specific even though it is less sensitive than the Berlin questionnaire. ${ }^{[21]}$ Another study carried out 


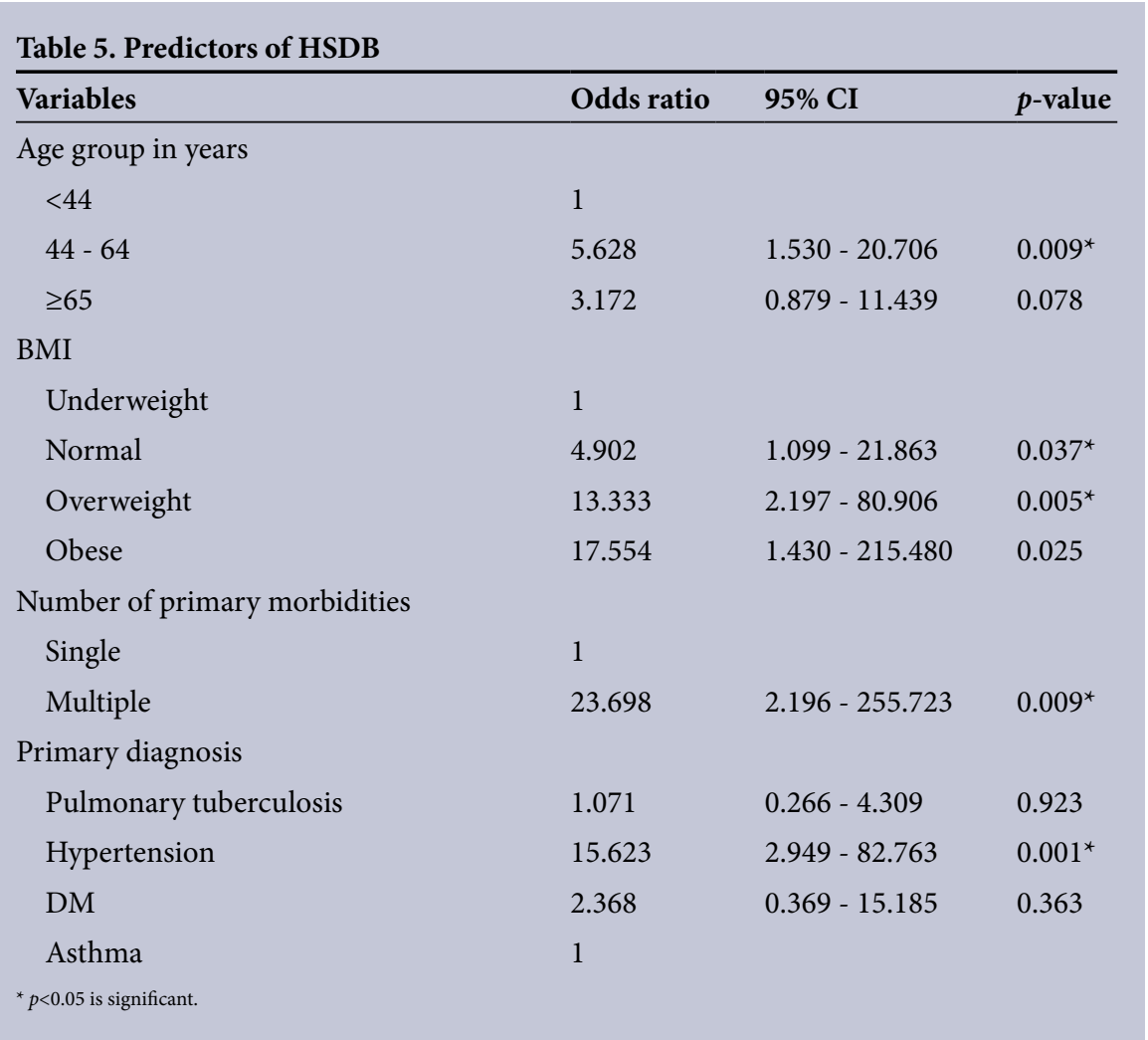

using the STOPBANG questionnaire in a tertiary facility in Lagos, Nigeria, showed that $36.3 \%$ of the respondents had a high risk of OSAS while $24.4 \%$ had EDS using the ESS. ${ }^{[22]}$ The seemingly lower value that was observed in the Lagos study compared with ours may be explained by the heterogeneity of the patients used for their study, since recruitment was done from both medical and surgical departments while ours focused strictly on those with medical conditions.

There appeared to be a significant increased risk of OSAS among middle-aged respondents when compared with those who were elderly. Increasing BMI was associated with higher risk of OSAS in this study; there was a strong positive association between them, with a gradual increase from respondents that were underweight to those that were obese. This has also been documented by several other studies. ${ }^{[9,23,24]}$

The significant association between respondents who were hypertensive and at high risk of OSAS could result from the higher percentage of this condition among those who were overweight and obese, compared with those with normal BMI, leading to increased risk of cardiovascular diseases. Of note also is the fact that those with multiple morbidities had a higher risk of OSAS compared with those with a single morbidity. Hypertension and DM combined constituted the highest risk, possibly resulting from the synergistic effect of these two conditions and their associations with obesity in the so-called metabolic syndrome. Asthma constituted the lowest risk as observed in this study. However, the percentage of those who were asthmatic among the respondents was small and may account for the low risk identified.

Several studies have described the increase in statistical significance of EDS and the risk of OSAS. ${ }^{[22,24]}$ Although there was a positive association between EDS (as measured by the ESS) and the risk of sleep apnoea (as assessed by the Berlin questionnaire), it was not significant in our study. This may result from the limitations of the instruments used in this study and the subjective nature of the responses obtained. An objective assessment of overnight pulse oximetry or PSG, which could be more informative, was not done in this study due to lack of availability and the huge cost implications.

Our study has shown a considerable risk of OSAS among patients attending the Medical Outpatients Department and its association with other health-related factors.
In conclusion, the risk of OSAS increased with increased BMI. Hypertension and the presence of multiple comorbid conditions also increased the risk of OSAS.

It is important that healthcare providers make efforts to screen all patients for OSAS using the available screening questionnaires designed for this purpose, and to ensure the prompt and comprehensive treatment of comorbidities. Increased awareness should be created among the healthcare workers with a view to asking questions regarding snoring and a tendency to fall asleep during the day. Attention should also be paid to preventive strategies, including lifestyle modifications.

Like other descriptive cross-sectional studies, recall bias of snoring while asleep or during daytime, especially when the respondent is not staying in the same room with the spouse, is a possible limitation. This limitation is minimised by the short recall period in the assessment tools. There is also the possibility of the respondents denying sleeping, either at work or while driving, for fear of losing their jobs.

We conclude that there is an urgent need for sleep laboratories in Nigeria so that patients with a high risk of OSAS can easily be referred for further evaluation.

\section{References}

1. Punjabi NM. The epidemiology of adult obstructive sleep apnea. Proc Am Thorac Soc 2008;5(2):136-143. [http://dx.doi.org/10.1513/pats.200709-155MG]

2. American Academy of Sleep Medicine. International Classification of Sleep Disorders, Third Edition (ICSD-3). Rochester, USA; 2014.

3. Akintunde AA, Okunola OO, Oluyombo R, Oladosu YO, Opadijo OG. Snoring and obstructive sleep apnea syndrome among hypertensive Nigerians: Prevalence and clinical correlates. Pan Afr Med J 2012;11:75.

4. Peppard PE, Young T, Palta M, Skatrud J. Prospective study of the association between sleep-disordered breathing and hypertension. N Engl J Med 2000;342(19):1378-1384. [http://dx.doi.org/10.1056/ NEJM200005113421901]

5. Peker Y, Carlson J, Hedner J. Increased incidence of coronary artery disease in sleep apnoea: A long-term follow-up. Eur Respir J 2006;28(3):596-602. [http:// dx.doi.org/10.1183/09031936.06.00107805]

6. Punjabi NM, Polotsky VY. Disorders of glucose metabolism in sleep apnea. J Appl Physiol 2005;99(5):1998-2007. [http://dx.doi.org/10.1152/ japplphysiol.00695.2005]

7. Khassawneh B, Ghazzawi M, Khader Y, et al. Symptoms and risk of obstructive sleep apnea in primary care patients in Jordan. Sleep Breath 2009;13(3):227-232. [http://dx.doi.org/10.1007/s11325-008-0240-4]

8. BaHammam AS, Alrajeh MS, Al-Jahdali $\mathrm{HH}$, BinSaeed AA. Prevalence of symptoms and risk of sleep apnea in middle-aged Saudi males in primary care. Saudi Med J 2008;29(3):423-426. 
9. Adewole OO, Adeyemo H, Ayeni F, et al. Prevalence and correlates of snoring among adults in Nigeria. Afr Health Sci 2008;8(2):108-113.

10. Abrishami A, Khajehdehi A, Chung F. A systematic review of screening questionnaires for obstructive sleep apnea. Can J Anaesth 2010;57(5):423-438. [http://dx.doi. org/10.1007/s12630-010-9280-x]

11. Senn O, Brack T, Russi EW, Bloch KE. A continuous positive airway pressure trial as a novel approach to the diagnosis of the obstructive sleep apnea syndrome. Chest 2006;129(1):67-75. [http://dx.doi.org/10.1378/chest.129.1.67]

12. Practice parameters for the indications for polysomnography and related procedures Polysomnography Task Force, American Sleep Disorders Association Standards of Practice Committee. Sleep 1997;20(6):406-422.

13. Pagel JF. Obstructive sleep apnea (OSA) in primary care: Evidence-based practice. J Am Board Fam Med 2007;20(4):392-398. [http://dx.doi.org/10.3122/ jabfm.2007.04.060201]

14. Young T, Evans L, Finn L, Palta M. Estimation of the clinically diagnosed proportion of sleep apnea syndrome in middle-aged men and women. Sleep 1997;20(9):705-706.

15. Sogebi OA, Ogunwale A. Risk factors of obstructive sleep apnea among Nigerian outpatients. Braz J Otorhinolaryngol 2012;78(6):27-33.

16. Hrubos-Strøm H, Randby A, Namtvedt SK, et al. A Norwegian population-based study on the risk and prevalence of obstructive sleep apnea. The Akershus Sleep Apnea Project (ASAP). J Sleep Res 2011;20(1 Pt 2):162-170. [http://dx.doi.org/10.1111/ j.1365-2869.2010.00861.x]
17. Chung F, Yegneswaran B, Liao P, et al. STOP questionnaire; a tool to screen patients with obstructive sleep apnea. Anesthesiology 2008;108(5):812-821. [http://dx.doi. org/10.1097/ALN.0b013e31816d83e4]

18. Netzer NC, Stoohs RA, Netzer CM, Clark K, Strohl KP. Using the Berlin Questionnaire to identify patients at risk for the sleep apnea syndrome. Ann Intern Med 1999;131(7):485-491.

19. Sleep-related breathing disorders in adults: recommendations for syndrome definition and measurement techniques in clinical research. The Report of an American Academy of Sleep Medicine Task Force. Sleep 1999;22(5):667-689.

20. Aigbokhaode A, Isara A. Obstructive sleep apnoea risk in patients attending medical outpatien clinicsin University of Benin Teaching Hospital, Benin City, Nigeria. S Afr Respir J2014;20(2):32-35

21. El-Sayed I. Comparison of four sleep questionnaires for screening obstructive sleep apnoea. Egyptian Journal of Chest Diseases and Tuberculosis 2012;61(4):433-441. [http://dx.doi.org/doi:10.1016/j.ejcdt.2012.07.003]

22. Ozoh OB, Okubadejo NU, Akinkugbe AO, et al. Prospective assessment of the risk of obstructive sleep apnea in patients attending a tertiary health facility in Sub-Saharan Africa. Pan Afr Med J 2014;17:302. [http://dx.doi.org/10.11604/pamj.2014.17.302.2898]

23. Hiestand DM, Britz P, Goldman M, Phillips B. Prevalence of symptoms and risk of sleep apnea in the US population: Results from the national sleep foundation: Sleep in America 2005 poll. Chest 2006;130(3):780-786. [http://dx.doi.org/10.1378/chest.130.3.780]

24. Sharma SK, Malik V, Vasudev C, et al. Prediction of obstructive sleep apnea in patients presenting to a tertiary care center. Sleep Breath 2006;10(3):147-154.

\section{The ESSENTIAL MEDICAL REFERENCE for every healthcare professional!}

The convenient pocket-sized design enables you to fit it comfortably into your hospital bag or coat pocket, so it can always be at hand for ready reference. South African Medicines Formulary (SAMF), produced by the Division of Clinical Pharmacology of the University of Cape Town, provides easy access to the latest, scientifically accurate information, including full drug profiles, clinical notes and special prescriber's points. The thoroughly updated 11th edition of SAMF is your essential reference to the rational, cost-effective and safe use of medicines.

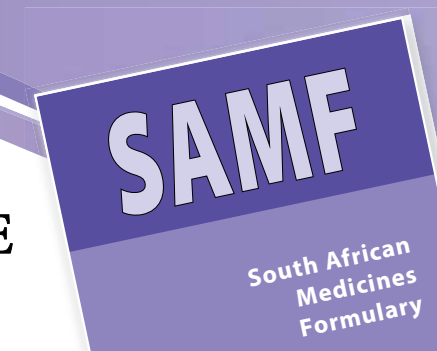

